\title{
Indicadores de sostenibilidad social y su relación con el concepto de capital social
}

\author{
Social sustainability indicators and their relationship with the concept of social capital
}

\section{Elquin Puentes}

Universidad Antonio Nariño. Bogotá (Colombia)

Facultad de Artes, Programa de Arquitectura

Vicerrectoría de Ciencia, Tecnología e Innovación

\section{Adriana Hidalgo-Guerrero}

Universidad Antonio Nariño. Bogotá (Colombia)

Facultad de Artes, Programa de Arquitectura

Vicerrectoría de Ciencia, Tecnología e Innovación

\section{Carolina Betancourt}

Universidad Antonio Nariño. Bogotá (Colombia)

Facultad de Artes, Programa de Arquitectura

Vicerrectoría de Ciencia, Tecnología e Innovación

\section{Yenny Ortiz-Bernal}

Universidad Antonio Nariño. Bogotá (Colombia)

Facultad de Artes, Programa de Arquitectura

Vicerrectoría de Ciencia, Tecnología e Innovación

\author{
Elquin Puentes \\ Arquitecto de la Universidad Nacional de Colombia. Bogotá (Colombia) \\ Doctor en urbanismo de la Universidad Politécnica de Madrid. Madrid (España) \\ Profesor e investigador de la Universidad Antonio Nariño. Bogotá (Colombia) \\ (D) https://orcid.org/0000-0003-4023-2829 \\ (10) elquinpuentes@uan.edu.co
}

\section{Adriana Hidalgo-Guerrero}

Arquitecta de la Universidad Nacional de Colombia. Bogotá (Colombia)

Doctora en urbanismo de la Universidad Politécnica de Madrid. Madrid (España)

Profesora e investigadora de la Universidad Antonio Nariño. Bogotá (Colombia)

https://scholar.google.com.co/citations?user=KYWnHcQAAAAJ\&hl=es

(D) http://orcid.org/0000-0003-2447-8423

(10) adrhidalgo@uan.edu.co

(10) adrhidalgoacademia@gmail.com

\section{Carolina Betancourt}

Arquitecta de la Universidad La Gran Colombia. Bogotá (Colombia)

Doctora en urbanismo de la UNAM. Ciudad de México (México)

Magister en arquitectura de la UNAM. Doctora en urbanismo de la UNAM. Ciudad de México (México)

Especialista en gestión urbana de la Universidad Piloto de Colombia. Bogotá (Colombia)

Profesora e investigadora de la Universidad Antonio Nariño. Bogotá (Colombia)

https://scholar.google.com/citations?user =x I6 IIUQAAAAJ\&hl=es

(1D) https://orcid.org/0000-000I-5706-4089

(10) carolina.betancourt@uan.edu.co.

Yenny Ortiz-Bernal

Arquitecta de la Universidad Antonio Nariño. Bogotá (Colombia)

Doctora en Urbanismo de la Universidad Politécnica de Madrid. Madrid (España)

Profesora e investigadora de la Universidad Antonio Nariño. Bogotá (Colombia)

https://scholar.google.com.co/citations?hl=es\&user=OFlfo9gAAAA]

(D) https://orcid.org/0000-0002-7252-4909

(19)yeortiz@uan.edu.co

(a) yennyorber@hotmail.fr

\section{Resumen}

Al hacer una revisión del estado del arte sobre el ámbito de la sostenibilidad social, se evidencian dos situaciones particulares: la primera es que la dimensión social de la sostenibilidad ha ido ganando importancia en las teorías y las políticas de desarrollo a escala mundial. La segunda es que, a pesar de lo anterior, la dimensión social de la sostenibilidad es la menos estudiada y la más difícil de medir, debido a la naturaleza y la complejidad de sus indicadores. El presente artículo propone una reflexión sobre estos mecanismos de medición, a fin de determinar qué indicadores del concepto capital social resultan de utilidad para la comprensión y la medición de la sostenibilidad social. Para ello se hace una revisión de la literatura que compara sistemas de indicadores aplicados en ambos conceptos. Dada la complejidad de dichos indicadores, el alcance del presente estudio se perfila como una serie de conclusiones derivadas de la reflexión sobre la complementariedad, las diferencias y las relaciones entre los sistemas de medición analizados, lo que resulta de potencial utilidad en la elaboración de indicadores concretos en estudios posteriores.

Palabras clave: Capital social; confianza; indicadores; sostenibilidad; sostenibilidad social; urbanismo

\begin{abstract}
When conducting a review of the state of the art in the field of social sustainability, two particular situations become evident: the first is that the social dimension of sustainability has been gaining importance in development theories and policies worldwide. The second is that, despite the above, the social dimension of sustainability is the least studied and the most difficult to measure due to the nature and complexity of its indicators. This article proposes a reflection on these measurement mechanisms, in order to determine which indicators of the social capital concept are useful for understanding and measuring social sustainability. To this end, a review of the literature is made, comparing indicator systems applied to both concepts. Given the complexity of these indicators, the scope of this study is outlined as a series of conclusions derived from the reflection on the complementarity, differences and relationships between the measurement systems analyzed, which is potentially useful in the elaboration of concrete indicators in subsequent studies.
\end{abstract}

Keywords: Social capital; trust; indicators; social sustainability; urban planning Evaluado: febrero 24 / 2020

\section{Introducción}

El presente artículo surge dentro del marco del proyecto de investigación titulado Formas de crecimiento y sostenibilidad en la vivienda social Latinoamericana. Estudio comparativo Bogotá-Buenos Aires-Fortaleza, el cual fue financiado por la Vicerrectoría de Ciencia, Tecnología e Innovación de la Universidad Antonio Nariño, mediante convocatoria interna para proyectos de investigación en 2017, e inscrito en el área institucional de Ciencias Humanas y Sociales, y realizado por integrantes del grupo de investigación Ciudad, Medio Ambiente y Hábitat Popular.

Al hacer una revisión de los principales sistemas de indicadores para la medición de la sostenibilidad social (SS) en el ámbito urbano, se hallaron elementos en común con los propuestos para la medición del capital social. De estas similitudes surge una cuestión central: ¿En qué medida estos sistemas de medición del capital social pueden complementar y aportar a los sistemas de medición de la sostenibilidad urbana, y en particular, la sostenibilidad social? Para despejar dicha cuestión, se propone revisar inicialmente la definición y los sistemas de medida de ambos conceptos, para luego hacer un ejercicio 
comparativo que llevará a la reflexión sobre los hallazgos que finalmente configuren algunas conclusiones, que, a su vez, son de potencial utilidad para estudios futuros que busquen definir indicadores concretos. Así pues, la hipótesis del presente artículo gira en torno a que los indicadores de capital social aportarían significativamente en conceptos y elementos de medida para la configuración de sistemas más efectivos y completos para la medición de la sostenibilidad urbana. Si bien en este documento se hace un acercamiento a algunos posibles indicadores, su alcance se enmarca dentro de una reflexión comparativa sobre la complementariedad, las similitudes y las diferencias de los indicadores estudiados, pues la producción de indicadores propiamente dicha requiere un estadio más avanzado que el aquí desarrollado, y relacionado con comprobaciones y el diseño aplicado, lo cual será tema de trabajos posteriores.

La SS es una de las tres dimensiones del desarrollo sostenible planteadas en el informe Brundtland (ONU, 1987), y es complementaria e inseparable de las dimensiones ambiental y económica. Si bien diversos autores aportan a la comprensión del concepto de SS, lo cierto es que no existe un consenso respecto a su significado (Bramley y Power, 2009; Martínez García et al., 2015). Aun así, el acercamiento a una serie de conceptos y palabras clave permite comprender mejor a qué se refiere la SS. A continuación se hará referencia a algunos de las más relevantes.

La dimensión social del desarrollo sostenible se fundamenta en las relaciones entre las personas, sus formas de organización, sus interacciones, la participación en la toma de decisiones y la distribución o la redistribución de los beneficios del desarrollo; a su vez, es una dimensión asociada a lo político-institucional (Parada-Zuluaga y Sánchez-Vásquez, 2014). A partir de una extensa revisión de literatura, Vallance et al. (2011) plantean tres aspectos imprescindibles en la comprensión del concepto de SS: 1) desarroIlo, que se refiere a la satisfacción de necesidades básicas a fin de crear justicia y capital social; 2) el puente hacia la sostenibilidad, referido a los cambios de comportamiento necesarios para lograr los objetivos y 3) el mantenimiento o la preservación en el tiempo de aquello que es susceptible de ser sostenido. Los autores proponen los mencionados aspectos de la SS para explorar de qué manera las contradicciones y los complementos entre ellos impiden o promueven el desarrollo sostenible, y las perfilan sobre áreas urbanas en las cuales puede evidenciarse el sentido de estas ideas.

De la revisión de autores hecha por Martínez García et al. (2015), se constata que sobre SS hay escasa teoría; sin embargo, encuentran convergencia en la valoración de indicadores relacionados con "el Capital Social, el sentido comunitario, la seguridad y la equidad social" (párrafo 16). El capital social se dimensiona a través de las redes, la confianza y los individuos organizados en colectivos, lo que les significa beneficios mutuos. El sentido comunitario es comprendido como un sentimiento que liga al individuo a lo colectivo, su sentido de pertenencia, que, a su vez, depende de la confianza y de la interacción social y conduce a su identificación con el vecindario, lo cual se traduce en mejor calidad de vida. En el mismo sentido, la equidad social también es un indicador de SS, y está fundamentada en la justicia social, el acceso a los recursos en condiciones de igualdad, la accesibilidad para la obtención de bienes y servicios, recursos, actividades e información, entre otros. Por su parte, Bramley y Power (2009) proponen dos dimensiones fundamentales para entender el concepto de SS: 1) la equidad social y 2) la sostenibilidad de la comunidad. Para otros,

\footnotetext{
[...] la sostenibilidad social se ha enfocado esencialmente, pero no siempre conscientemente, desde el punto de vista de la geografía, como una búsqueda de la equidad espacial. La definición de equidad espacial se refiere a la equidad, es decir la distribución justa (no siempre igual) de la riqueza en un territorio [...] La equidad espacial es solo una parte de la sostenibilidad social, y probablemente pequeña, pero es una parte sólida, con propuestas concretas de acción e impactos concretos a escala local y regional. (Pitarch-Garrido, 2018, ítem. 2.1 párrafo 6)
}

De acuerdo con lo anterior, los desafíos de SS son más agudos mientras más marginada o excluida sea la población (Kohon, 2018a; Kohon, 2018b); dicho en otras palabras, en los contextos de pobreza e inequidad (Lara-Hernández y Melis, 2018) se acercan a las ideas de Maslow, en el sentido de que la satisfacción de necesidades básicas conlleva posteriores beneficios para el medio ambiente $y$, por otra parte, hacen una crítica al concepto de SS, en la medida en que se la asocia a crear conciencia social, más que a pensar en la sostenibilidad del tejido social.

El panorama está cambiando en el mundo, y los gobiernos, los arquitectos, los planificadores y los organismos internacionales son cada vez más los interesados en avanzar en el tema de la SS (Woodcraft, 2015); entre otras cosas, porque se considera que el desarrollo sin equidad no puede considerarse desarrollo (Pitarch-Garrido, 2018). La SS es, sin embargo, el ámbito que se encuentra en menor grado de desarrollo en relación con los demás que conforman el desarrollo sostenible. 
Si pudiéramos identificar las variables que inciden en los indicadores de SS, una de las más relevantes sería la de la integración social, la cual debería ser el pilar de las políticas públicas. Colantonio (2007) y Martínez García et al. (2015), por su parte, sostienen que los indicadores de SS miden aspectos más cualitativos e indefinidos, lo cual puede ser la causa de la complejidad en sus sistemas de medición y de análisis. Así mismo, Woodcraft (2015) asegura:

It has developed over a number of years in response to the dominance of environmental concerns and technological solutions in urban development and the lack of progress in tackling social issues in cities such as inequality, displacement, liveability and the increasing need for affordable housing. (pp. 133)

\section{En la misma línea, Kohon (2018a) señala que}

El concepto de sostenibilidad, ha sido adoptado en el urbanismo tanto en la teoría como en la práctica, pero la dimensión social de la sostenibilidad es la menos desarrollada y la más subvalorada, a pesar de que conceptualmente y de manera práctica, esta dimensión ofrece soluciones a aspectos de sostenibilidad y problemas urbanos estructurales. (p. 4)

\section{Indicadores de sostenibilidad social}

Respecto a la medición de la SS, se hará seguidamente una revisión teórica que permita tener una idea general de los elementos utilizados para determinar su presencia, para luego compararlos con los planteados para medir el capital social. Las políticas de desarrollo sostenible se enmarcan dentro de tres dimensiones: la económica, la ambiental y la social, que son sus pilares (Naciones Unidas, 2005). Este ha sido un tema de debate que ha venido nutriéndose, y cada vez son más acotados los diseños de los indicadores y los lineamientos para su respectiva evaluación y su medición; sin embargo, la medición de la dimensión social de la sostenibilidad se configura como el aspecto menos desarrollado en lo que a indicadores se refiere (Martínez García et al., 2015; Serrano, 2009), dado que se sustenta en modelos cualitativos. Esta dimensión, como se mencionaba en el apartado anterior, se basa, entre otros conceptos, en el mantenimiento de la cohesión social y en la capacidad de los grupos humanos para trabajar en la consecución de fines de bien común. Para medir este ámbito de la sostenibilidad se han desarrollado diversos sistemas de indicadores: la Comisión de Desarrollo Sustentable de Naciones Unidas elaboró, por ejemplo, un esquema metodológico para cada indicador, que está basado en el esquema presión-estado-respuesta (PER). Dicho sistema no es específico para la dimensión social, por cuanto se basa en la interrelación entre la presión que ejercen las actividades humanas sobre los recursos naturales, que afectan su estado, y luego, las medidas que esas mismos grupos humanos ela- boran como respuesta. Según Gallopin (2006), ni este modelo ni sus variantes toman en cuenta todas las dimensiones a la vez, sino que, paradójicamente, al hacer énfasis de manera cuantitativa en los efectos producidos por el ser humano sobre los recursos naturales, pierden de vista el sentido social de la sostenibilidad.

La dimensión social se desarrolla a partir de indicadores compuestos, tales como la longevidad, la alfabetización y el nivel de vida. Tal es el caso para el índice de desarrollo humano (IDH), establecido en 1990 por el Programa de las Naciones Unidas para el Desarrollo (PNUD) (Montserrat Casado, 1996), y el cual señala que dicho índice mide el desarrollo humano desde un enfoque multidimensional, donde lo económico es sustancial, pero no suficiente; es decir, el desarrollo económico es importante sin que eso vaya en detrimento de la calidad de vida de los seres humanos; sin embargo, y a pesar de su planteamiento, el índice ha sido criticado ampliamente, debido a que en sus inicios no hacía referencia a los Derechos Humanos (DD. $\mathrm{HH}$.) ni a temas relacionados con el medio ambiente.

En la misma línea tenemos el llamado índice de pobreza humana, el cual propone la medición de la pobreza a partir de aspectos como el acceso a los conocimientos, la morbilidad y el acceso a condiciones de vida aceptables. Dichos aspectos, a su vez, son calculados por medio de indicadores sociales como el porcentaje de personas que no sobrepasan los 40 años, la tasa de analfabetismo, el porcentaje de personas sin acceso a agua apta para el consumo humano, o sin oferta de salud, el porcentaje de niños con desnutrición, etc. Al revisar la lista de indicadores relacionados con el aspecto social de la sostenibilidad, propuesta por las Naciones Unidas en 2011, se hace evidente que las cifras apuntan a datos puramente cuantitativos, pero no existen datos que busquen dimensionar las cualidades de estos. De los diferentes grupos de indicadores (salud, educación, población, equidad, vivienda y seguridad), el más relacionado con el ámbito social es el de la equidad; sin embargo, y por tomar un ejemplo, el indicador de "igualdad de género" se mide apelando a la diferencia del promedio salarial entre hombres y mujeres, sin tener en cuenta otras dimensiones de igualdad, tales como la participación en política, la participación en cargos públicos, la discriminación laboral, etc.

\section{Metodología}

Para lograr los objetivos del presente artículo, se proponen las siguientes fases metodológicas:

1) Fase I, que se inicia con la revisión del estado del arte de los dos conceptos que configuran 
el estudio: la SS y el capital social; para el primero se toman como referencia los avances hechos en el proyecto de investigación Formas de crecimiento y sostenibilidad en la vivienda social Latinoamericana. Estudio comparativo Bogotá-Buenos Aires-Fortaleza, cuyo objetivo era determinar la aplicabilidad de indicadores de sostenibilidad urbana en contextos de ciudades latinoamericanas. En dicho proceso se complementaron los indicadores propuestos por la Agencia de Ecología Urbana de Barcelona con otras fuentes documentales que aportaron elementos diversos y complementarios sobre el concepto de SS en particular, y que se consignan en la tabla 1 . Por otra parte, se revisaron definiciones y sistemas de medida del capital social derivados de varias fuentes: estudios comparativos realizados, exploraciones en motores de búsqueda, como Vosviewer, y revisiones de autores fundamentales, como Robert Putnam, Pierre Bourdieu y Francis Fukuyama.

2) Fase II: A partir de la revisión llevada a cabo, se elabora un ejercicio comparativo que permite registrar las similitudes, las diferencias y las complementariedades de los sistemas de medida estudiados, lo cual se consigna en la tabla 2.

3) Fase III, en la cual se determinan las particularidades y las tendencias entre los diferentes autores y los sistemas de medición, de manera que se configuran los resultados que permiten hacer una reflexión comparativa y producir las conclusiones que configuran la fase IV. indicadores de SS.

Fuente: elaboración propia (2019). CC BY NC
Saneamiento básico, acceso seguro a agua potable, esperanza de vida al nacer, tasa de mortalidad infantil, esquema básico de vacunación, planificación familiar, gasto ejercido del sector salud, educación básica, tasa bruta de matrícula escolar en educación básica, tasa de alfabetización, educación media superior, educación superior, mujeres $\times 100$ hombres PEA, matrícula escolar femenina y masculina, tasa de crecimiento de la población, tasa de migración, densidad de población, igualdad de género, tasa de desempleo, índice general de pobreza, índice de GINI, condiciones de vivienda, crimen.
Comisión de Desarrollo Sostenible (CDS) Naciones Unidas (SIGFRE-DRS) (2019).

Sachs (1999)

Polèse y Stren (2000); Putnam (2000)

Sociedad civil, diversidad cultural y social, desarrollo (o crecimiento) compatible, fomento de un entorno propicio para la cohabitación compatible de grupos cultural y socialmente diversos.

Equidad, democracia, DD. HH., homogeneidad social, distribución equitativa del ingreso empleo, acceso equitativo a los recursos sociales, servicios.
Cuantitativo

Mixto

\section{Cualitativo}

Mixto

Sustento, equidad, capacidad para soportar presiones externas, redes de seguridad.

Cuantitativo

DFID (1999), citado por Martínez

García et al. (2015)

Inclusión, equidad, pobreza, medios de subsistencia.

Hans-Böckler-Stiftung (2001), citado por Martínez García et al. (2015)

Trabajo remunerado y voluntario, necesidades básicas, seguridad social, igualdad de oportunidades para participar en una sociedad democrática, habilitación de innovación social.

Cuantitativo

Thin et al. (2002); DIFD, citado por Martínez García et al. (2015)

Justicia social, solidaridad, participación, seguridad.

Mixto

Omann y Spangenberg (2002), citados por Martínez García et al. (2015)

Educación, habilidades, experiencia, consumo, ingresos, empleo, participación.

Cuantitativo

Necesidades básicas, discapacidad personal, necesidades de las generaciones futuras, capital social, equidad, diversidad cultural y comunitaria, empoderamiento y participación.

Mixto (2004)

Bramley et al. (2006)

Interacciones en la comunidad/social, redes, participación comunitaria, orgullo y sentido del lugar, estabilidad en la comunidad, seguridad.

Cualitativo

Equidad social y la sostenibilidad de las comunidades, que incluye el acceso a servicios y oportunidades, mientras que la sostenibilidad de las comunidades incluye varias subdimensiones, tales como el apego al barrio, la interacción social, la seguridad en el Mixto

Dempsey et al. (2012) barrio, la calidad percibida del entorno local, la satisfacción con la casa, la estabilidad y la participación en actividades cívicas colectivas.

Dave Seema (2011)

Equidad social, cohesión social, y el capital social, la salud y el bienestar de los residentes.

Cualitativo
Mixto 


\section{Resultados}

Al elaborar la revisión del estado del arte sobre la cuestión, podemos observar la diferencia de enfoques entre los planteamientos realizados por teóricos del tema y los sistemas de medición utilizados por organismos multilaterales. En el primer caso se observan conceptos de SS como: equidad, homogeneidad social, acceso equitativo a los recursos sociales (Sachs, 1999); inclusión, medios de subsistencia (DDIF, 1999, citado por Martínez et al., 2015); oportunidades de participación democrática en igualdad de condiciones (Hans-Böckler-Stiftung, 2001, citado por Martínez García et al., 2015), y capital social, diversidad cultural, empoderamiento y participación (Baines y Morgan, 2004). En el caso de los instrumentos implementados por PNUD, como el sistema PER o el índice de pobreza humana, se observa una tendencia a indicadores exclusivamente cuantificables, y no se incluye ningún indicador que no esté relacionado con los conceptos ya mencionados, como se muestra en la tabla 1.

Una observación que surge de la revisión mostrada en la tabla 1 es que se presenta falta de coherencia entre las teorías y los sistemas utilizados para medir la SS en términos prácticos. Dos ideas propuestas por Gallopin (2006) pueden ayudar a entender la situación: 1) en los instrumentos utilizados para la evaluación de la SS hay escasez de metodologías, pues en la práctica dicha evaluación se realiza mediante los impactos sociales, vistos a través de variables económicas, o mediante la redefinición de "medio ambiente", lo cual amplía la cobertura temática de la evaluación. Por otra parte, 2) el desarrollo de nuevos indicadores de sostenibilidad se concentra más en el cálculo de nuevos conceptos, en vez de mejorar la evaluación de conceptos más tradicionales, como, por ejemplo, el de equidad; de hecho, esta última se mide en términos de distribución del ingreso y otras variables monetarias, y se aparta, por tanto, del sentido holístico de la SS. Tal situación hace evidente que las herramientas utilizadas por organismos multilaterales - y en general, por los gobiernos - para calcular el grado de SS carecen de precisión en cuanto sus medidas, y solo hacen una aproximación tangencial a la situación.

En este punto surge una cuestión que afecta de igual manera al concepto del capital social, y aunque resulta contradictoria en sí misma, vale la pena abordarla: ¿es posible hacer más cuantificables los indicadores cualitativos? Y la respuesta pasa por hacer un ejercicio que permita contabilizar elementos normalmente intangibles, desarrollando, como lo plantea Gallopin (2006), metodologías que, además de las variables monetarias, tengan en cuenta variables que se mueven más en el ámbito de lo cualitativo y, en ocasiones, de lo subjetivo.
En tal sentido, Sudarsky (2001) propone y hace para Colombia una medición de capital social donde se calculan indicadores lógicamente cuantitativos, y otros tan subjetivos como la confianza en las instituciones o la confianza en los vecinos, recurriendo a métodos que permiten cuantificarlos de manera estadística y precisa.

De la misma forma, indicadores como el arraigo al barrio, la calidad percibida del entorno local y la satisfacción con la casa, propuestos por Dempsey (2012); el orgullo y el sentido del lugar, y la estabilidad en la comunidad, de Brambley et al. (2006), como se muestra en la tabla 1, son todos indicadores de SS, y si bien complejos de medir, no son menos importantes, y requieren el diseño de herramientas para ser incluidos en los diagnósticos que permitan configurar estrategias de acción en busca de una SS entendida de manera integral.

\section{Discusión}

\section{Relación entre indicadores de sostenibilidad social y capital social}

A fin de relacionar los indicadores de SS con los propuestos para medir el capital social, se revisarán a continuación este último concepto y sus componentes. El término se viene usando desde principios del siglo $X X$, inicialmente en pedagogía, y posteriormente en temas asociados a teorías de desarrollo económico de la década de 1970, pero fue en la de 1980 cuando el concepto se afianzó, al ser utilizado en sociología. En este último campo destacan los estudios de Colantonio (2007), en modelos de economía alternativa y de teorías de desarrollo, así como los de Bourdieu (2008), Putnam et al. (1994) y Ostrom y Ahn (2003).

Al igual que lo que pasa con la definición de SS, en los mencionados aportes no se cuenta con un consenso sobre la definición de capital social. Este concepto surge de realizar una analogía con el concepto de capital económico, y ha sido utilizado en diversas disciplinas, tales como la pedagogía, la sociología, la planificación espacial y la economía, entre otras, razón por la cual su sentido y sus indicadores tienden a complejizarse y a particularizarse de acuerdo con la disciplina a la que se estén aplicando. Por ejemplo, desde el ámbito de la sociología Woolcock y Narayan (2000) lo definen como la cantidad de recursos tanto reales como intangibles que pueden ser acumulados por un individuo, por el hecho de pertenecer a una red de relaciones basada en la familiaridad y el reconocimiento de sus miembros. Por otra parte, Robert Putnam et al. (1994) fue el primero en relacio- 
(1) Tabla 2. tabla comparativa entre grupos de indicadores de capital social, y de SS.

Fuente: elaboración propia (2019). CC BY NC nar el concepto de capital social a un ámbito territorial; en su investigación, demuestra que conceptos como el cumplimiento de normas y la confianza están directamente relacionados con los niveles de riqueza y bienestar presentes en las regiones del sur y el centro de Italia, lo cual determina la importancia del capital social en la calidad de vida de los grupos humanos. Francis Fukuyama (2011), por su parte, hace énfasis en el concepto de confianza como principio estructural del capital social, y asegura que en las sociedades donde no existe confianza entre sus miembros es muy difícil que el capital social pueda ser instaurado de manera efectiva en planes y políticas.

En el ámbito latinoamericano, Sudarsky (2001) propone una definición que se basa, según él, en una "intuición presente en la mayoría de las personas, y que supone que las relaciones sociales son de utilidad para lograr objetivos, que sin estas no sería posible conseguir".

A continuación se hace una síntesis de los componentes del capital social, y se los compara con los de SS. Para esta comparación, se toma como referencia la clasificación elaborada por Puentes (2015), en la que se proponen los siguientes grupos de indicadores de capital social: grupos y organizaciones; redes, normas y acuerdos; confianza y solidaridad; empoderamiento institucional; calidad de vida y tradición. Luego se incluyen los conceptos hallados que se relacionen con la SS, y se los ubica en el mismo sistema de clasificación ya propuesto, para poder determinar coincidencias y diferencias. De esa manera, se observa que de los 22 conceptos revisados, 3 de ellos están relacionados con la categoría de grupos; otros 2, con la de redes; uno, con normas y acuerdos; 3 , con empoderamiento institucional; 4 , con mejoras en la calidad de vida, y 5 más, con la categoría de tradición y saber local. Los 4 restantes requieren la aparición de una nueva categoría: la equidad, como se muestra en la tabla 2.

Los indicadores de desarrollo sostenible propuestos por las Naciones Unidas y relacionados con la SS se clasifican en dos ámbitos: el primero es el de equidad, que comprende aspectos como la igualdad de género, la tasa de desempleo, el índice de pobreza y el índice de GINI. El segundo el de la seguridad. En ambos se evidencia que los elementos de medida propuestos se relacionan con valores que no permiten medir elementos estructurales de la SS revisados en el presente trabajo, tales como el nivel de cohesión social o el nivel de equidad.

$\begin{array}{ccc}\text { Organizaciones y grupos } & \text { Instrumentos de información y } \\ \text { comunitarios } & \text { comunicación } & \text { Acuerdos de funcionamiento }\end{array}$

\begin{tabular}{|c|c|c|c|c|}
\hline \multirow[t]{2}{*}{$\begin{array}{l}\text { Indicadores de } \\
\text { capital social }\end{array}$} & Membresía & $\begin{array}{l}\text { Eficacia de los canales de } \\
\text { participación establecidos }\end{array}$ & Métodos de toma de decisiones & Confianza en las instituciones \\
\hline & Heterogeneidad & & $\begin{array}{l}\text { Elección de líderes y } \\
\text { representantes }\end{array}$ & El bien común como prioridad \\
\hline
\end{tabular}

Grado de solidaridad

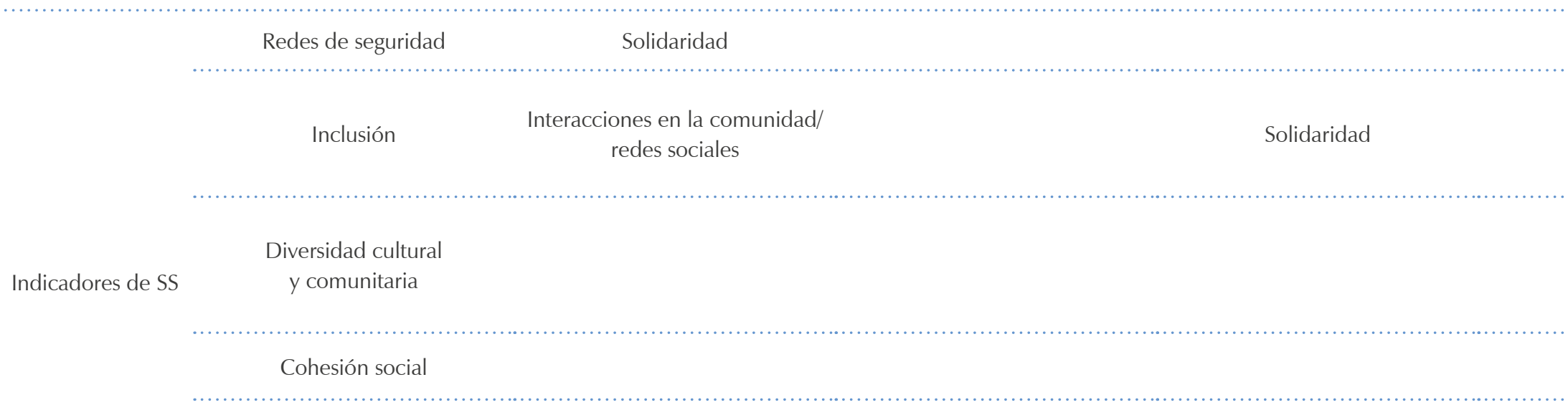




\section{Conclusiones}

A partir de la revisión realizada, se hace evidente que los sistemas utilizados para medir la SS urbana se limitan a una visión instrumentalista, en la medida en que se concentran en determinar la presencia cuantitativa de dichos sistemas, pero no permiten determinar de manera clara su efectividad ni su alcance, en términos de cohesión social, equidad o confianza, tres conceptos estructurales de ese ámbito de la sostenibilidad, y también identificados en el presente trabajo.

Es así como los indicadores propuestos, por ejemplo, por las Naciones Unidas para medir la equidad (la igualdad de género, la tasa de desempleo, el índice de pobreza y el índice de GINI) si bien son de gran utilidad para detectar las problemáticas específicamente en cifras, no permiten detectar otras dimensiones. Estas variables de medida son útiles, por ejemplo, para determinar la cantidad de desempleados en cierto grupo humano, pero no permiten determinar las condiciones del empleo existente, ni si estas implican equidad en un sentido amplio.

Otro caso es el de la variable denominada "igualdad de género", la cual se limita a determinar las diferencias salariales entre hombres y mujeres, pero no entra a analizar otros aspectos relacionados con el tema de inclusión, como el de la participación de minorías en política, por poner un ejemplo.

Lo anterior pone de manifiesto otro aspecto crítico en la elaboración de indicadores sociales para un grupo humano: la necesidad de elaborar y aplicar sistemas de indicadores ajustados a los contextos particulares. Hay comunidades para las cuales unos indicadores pueden representar un matiz diferencial, y eso es digno de tenerse en cuenta: por ejemplo, la heterogeneidad en la composición de organizaciones y grupos, propuesta por autores como Putnam (1994), puede representar una gran diferencia si hablamos de una comunidad urbana o de una comunidad rural.

La medición de indicadores sociales presenta un nivel de complejidad alto, y por ello se hace necesario desarrollar la investigación y la implementación de los avances realizados en contextos nacionales y regionales específicos. Un ejemplo de sistema de medición de capital social para Colombia es el propuesto e implementado por Sudarsky (2001), el cual es el más elaborado en el contexto colombiano hasta 2014; sin embargo, al compararlo con los sistemas de medición utilizados por organismos multilaterales se hace evidente que no han sido tomados en cuenta

\begin{tabular}{|c|c|c|c|}
\hline $\begin{array}{l}\text { Empoderamiento } \\
\text { institucional }\end{array}$ & Calidad de vida & Tradición y saber local & Equidad \\
\hline $\begin{array}{c}\text { Participación de las } \\
\text { comunidades en decisiones } \\
\text { políticas }\end{array}$ & $\begin{array}{c}\text { Mejoras en la calidad de } \\
\text { vida derivadas de la relación } \\
\text { entre los miembros y la } \\
\text { autoorganización }\end{array}$ & $\begin{array}{l}\text { Elementos de tradición } \\
\text { aplicados a procesos }\end{array}$ & \\
\hline $\begin{array}{l}\text { Conocimiento y uso de } \\
\text { instrumentos democráticos de } \\
\text { participación }\end{array}$ & & & \\
\hline
\end{tabular}

Participación

Acceso a servicios básicos,

acceso a salud, morbilidad, nivel de educación, nivel de alfabetización

\section{Capacidad para soportar} presiones externas
Equidad en la distribución equitativa del ingreso, empleo, acceso equitativo a los recursos sociales

Igualdad de oportunidades para participar en una sociedad democrática, habilitación de innovación social

Medios de subsistencia Trabajo remunerado voluntario

Habilitación de innovación social

Discapacidad personal

Orgullo y sentido del lugar

Apego al barrio 
sus aportes. De igual forma, en el caso de la SS se hace necesario un sistema de medición de la sostenibilidad urbana específica que incluya las particularidades de las ciudades y del territorio.

En este sentido, el presente documento pone de manifiesto un tema que vale la pena profundizar: algunos indicadores utilizados en capital social son complementarios en la medición de la SS, pues aportan elementos de un tipo más cuantitativo que, sin embargo, brinda información respecto a la calidad de los indicadores. Por ejemplo, en el tema de redes no solo se habla del número existente de redes en un grupo humano, sino que se habla de grupos inclusivos, y ello implica otro tipo de información, como lo es la composición de dichos grupos, su heterogeneidad racial, de género y de condición económica, su nivel de participación, etc. Se hacen necesa- rios sistemas de indicadores de SS que incluyan de manera más decidida temas que no solo estén relacionados con elementos cuantitativos, sino que puedan incluirse otras dimensiones, y que, a pesar de su dificultad para medirse, sean tenidos en cuenta como elementos indicativos estructurales de la cohesión social y el bienestar integral de los grupos humanos.

Lo hallado en la revisión aquí llevada a cabo pone de manifiesto que el concepto de capital social y sus sistemas de medida sí pueden aportar de manera efectiva en la medición de la SS, pero, además, se hace evidente otra situación: ambos conceptos y sus indicadores presentan limitaciones instrumentales similares y que requieren la elaboración sistemática de metodologías que permitan su medición y su adaptación a contextos particulares.

\section{Referencias}

Baines, J. y Morgan B. (2014). Sustainability appraisal: A social perspective. En B. DalalClayton y B. Sadler (Eds.), Sustainability appraisal. A review of international experience and practice. International Institute for Environment and Development.

Bourdieu, P. (2008). El sentido práctico. Siglo XXI Editores.

Bramley, G., Dempsey, N., Power, S. y Brown, C. (2006). What is 'social sustainability', and how do our existing urban forms perform in nurturing it?

https://www.researchgate.net/ publication/312913452 What is sustainability and how do existing urban forms perform_in_nurturing_it

Bramley, G. y Power, S. (2009). Urban form and social sustainability: The role of density and housing type. Environment and Planning $B$, 36(1).

https://doi.org/doi:10.1068/b33129.

Chambers, R. y Conway, G. (1992). Sustainable rural livelihoods: Practical concepts for the 21st Century, IDS Discussion Paper 296. IDS.

Colantonio, A. (2007). Social sustainability: An exploratory analysis of its definition, assessment methods metrics and toolstitle. 01. EIBURS Working Paper Series. Oxford. http://eprints.Ise.ac.uk/35947/.

Dempsey, N., Branley, G., Power, S. y Brown, C. (2012). The social dimension of sustainable development: Defining urban social sustainability. Sustainable Development, 19(5), 289-300.

Fukuyama, F. (2011). Capital social y desarrollo, la agenda venidera. Comisión económica para América Latina y el Caribe. Libros CEPAL 71, Cap. II, pp. 33-40. Santiago de Chile.

Gallopin, G. (2006). Los indicadores de desarrollo sostenible: aspectos conceptuales y metodológicos. Seminario de Expertos sobre indicadores de sostenibilidad en la formulación y seguimiento de políticas (4-6 de octubre, 2006, Santiago) Memorias. Santiago de Chile.

Kohon, J. (2018a). Interpreting the social dimension of sustainability: Connecting theory and community planning practice with a social determinants of health framework. The International Journal of Sustainability in Economic, Social, and Cultural Context 14 (3), 1-19. https://doi.org/https://doi.org/10.1016/j. cCs.2018.08.005.
Kohon, J. (2018b). Social inclusion in the sustainable neighborhood? Idealism of urban social sustainability theory complicated by realities of community planning practice. City, Culture and Society 15, 14-22. https://doi.org/https://doi.org/10.1016/j. CCS.2018.08.005.

Langebeck Cuéllar, E. y Beltrán Vargas, J.E. (2016). Sustentabilidad territorial de los procesos de ocupación urbano-rurales en Usme, Bogotá - Colombia. Gestión y Ambiente 19(1), 11-30.

Lara-Hernández, J. y Melis, A. (2018). Understanding the temporary appropriation in relationship to social sustainability. Sustainable Cities and Society 39. https://doi.org/10.1016/j.scs.2018.03.004.

Martínez García, E., Baeriswyl Rada, E. y Fuentes P. (2015). Análisis de la sostenibilidad social en tipologías residenciales del Gran Concepción (Chile). Cuaderno Urbano 19. https://dialnet.unirioja.es/servlet/ articulo? codigo $=5960754$

Montserrat Casado, F. (1996). El desarrollo como proceso multidimensional: El IDH en 1995. Boletín Económico de ICE Información Comercial Española, n. 2492.

Naciones Unidas. (2005). Documento final de la Cumbre Mundial 2005. Asamblea General de las Naciones Unidas, 24 de octubre.

ONU. (1987). Informe Brundtland. Nueva York.

Ostrom, E. y Ahn, T. (2003). Una perspectiva del capital social desde las ciencias sociales: Capital social y acción colectiva. Revista Mexicana de Sociología 65(1), 155-233.

Parada-Zuluaga, L. y Sánchez-Vásquez, L. (2014). Desarrollo sostenible en Colombia una utopía, una necesidad del presente $y$ un alivio para el futuro. Contexto 3(1). http://revistas.ugca.edu.co/index.php/ contexto/article/view/288/427.

Pitarch-Garrido, M. (2018). Social sustainability in metropolitan areas: Accessibility and equity in the case of the metropolitan area of Valencia (Spain). Sustainability 10(2). https://doi.org/10.3390/su10020371.

Polèse, M. y Stern, R. (2000). The social sustainability of cities: Diversity and the management of change. University of Toronto Press.

Puentes, E. (2015). Mejoramiento integral de barrios y capital social. (Monografía) Universidad Politécnica de Madrid.
Putnam, R. (2000). Bowling alone. Simon and Schuster.

Putnam, R., Leonardi,L. y Nanetti, R. (1994). Making democracy work: Civic traditions in modern Italy. Princeton University Press.

Sachs, I. (1999). Social sustainability and whole development: Exploring the dimensions of sustainable development. En B. Egon y J. Tomas (Eds.), Sustainability and the social sciences: a crossdisciplinary approach to integrating environmental considerations into theoretical reorientation (pp. 304-336). Zed Books.

Seema, D. (2011). Neibhbourhood density and social sustainability in cities of developing countries. Sustainable Development, 19, 189-205.

Serrano, J. M. (2009). La dimensión social de desarrollo sostenible: Análisis conceptual y representación espacial [The Socialdimensión of Sustainable Development: Conceptual Analysis and Spatial Characterisation]. Universidad de Alcalá.

Sinner, J., Baines, J., Crengle, H., Salmon, G., Fenemor, A. y Tipa, G. (2004). Sustainable Development: A summary of key concepts. Ecologic Research Report No. 2. www.ecologic.org.nz.

Sistema de Información Geográfica de Fuentes Renovables de Energía para la Planeación del Desarrollo Regional Sustentable (SIGFRE-DRS). (2019) Desarrollo sustentable. http://energia.ugto.mx/index.php/ desarrollo-sustentable.

Sudarsky, J. (2001). El capital social de Colombia. Departamento Nacional de Planeación.

Vallance, S., Perkins, H., y Dixon, J. (2011). What is social sustainability? A clarification of concepts. Geoforum 42(3). https://doi.org/https://doi.org/10.1016/j. geoforum.2011.01.002.

Woodcraft, S. (2015). Understanding and measuring social sustainability. Journal of Urban Regeneration \& Renewal 8(2), 133-144

Woolcock, M. y Narayan D. (2000). Capital social: Implicaciones para la teoría, la investigación y las políticas sobre desarrollo. World Bank Research Observer 15(2), 225-249. 
A Portada: Catedral de Notre Dame de Paris durante los trabajos de reparación en octubre de 2020 Fotografía: Arquitecto Andrés Avila Gómez (octubre, 2020)
(A) Orientación editorial

\section{Enfoque y alcance}

La Revista de Arquitectura (Bogotá) ( (ISSN 1657-0308 Impresa y E-ISSN 2357-626X en línea) es una publicación científica seriada de acceso abierto, arbitrada mediante revisión por pares (doble ciego) e indexada, en donde se publican resultados de investigación originales e inéditos.

Está dirigida a la comunidad académica y profesional de las áreas afines a la disciplina. Es editada por la Facultad de Diseño y el Centro de Investigaciones (CIFAR) de la Universidad Católica de Colombia en Bogotá (Colombia).

La principal área científica a la que se adscribe la Revista de Arquitectura (Bogotá) según la OCDE es:

Gran área: 6. Humanidades

Área: 6.D. Arte

Disciplina: 6D07. Arquitectura y Urbanismo

También se publican artículos de las disciplinas como 2A02, Ingeniería arquitectónica; 5G03, Estudios urbanos (planificación y desarrollo); 6D07, Diseño.

Los objetivos de la Revista de Arquitectura (Bogotá) son:

- Promover la divulgación y difusión del conocimiento generado a nivel local, nacional e internacional

- Conformar un espacio para la construcción de comunidades académicas y la discusión en torno a las secciones definidas.

- Fomentar la diversidad institucional y geográfica de los autores que participan en la publicación.

- Potenciar la discusión de experiencias e intercambios científicos entre investigadores y profesionales.

- Contribuir a la visión integral de la arquitectura, por medio de la concurrencia y articulación de las secciones mediante la publicación de artículos de calidad.

- Publicar artículos originales e inéditos que han pasado por revisión de pares, para asegurar que se cumplen las normas éticas, de calidad, validez científica, editorial e investigativa.

- Fomentar la divulgación de las investigaciones y actividades desarrolladas en la Universidad Católica de Colombia.
Palabras clave de la Revista de Arquitectura (Bogotá): arquitectura, diseño, educación arquitectónica, proyecto y construcción, urbanismo.

Idiomas de publicación: español, inglés, portugués y francés.

Título abreviado: Rev. Arquit.

Titulo corto: RevArq

\section{Políticas de sección}

La revista se estructura en tres secciones correspondientes a las líneas de investigación activas y aprobadas por la institución, y dos complementarias, que presentan dinámicas propias de la Facultad de Diseño y las publicaciones relacionadas con la disciplina.

Cultura y espacio urbano. En esta sección se publican los artículos que se refieren a fenómenos sociales en relación con el espacio urbano, atendiendo aspectos de la historia, el patrimonio cultural y físico, y la estructura formal de las ciudades y el territorio.

Proyecto arquitectónico y urbano. En esta sección se presentan artículos sobre el concepto de proyecto entendido como elemento que define y orienta las condiciones proyectuales que devienen en los hechos arquitectónicos o urbanos, y la forma como estos se convierten en un proceso de investigación y nuevo de conocimiento. También se presentan proyectos que sean resultados de investigación, los cuales se validan por medio de la ejecución y transformación en obra construida del proceso investigativo. También se contempla la publicación de investigaciones relacionadas con la pedagogía y didáctica de la arquitectura, el urbanismo y el diseño.

Tecnología, medioambiente y sostenibilidad. En esta sección se presentan artículos acerca de sistemas estructurales, materiales y procesos constructivos, medioambiente y gestión, relacionados con los entornos social-cultural, ecológico y económico.

Desde la Facultad. En esta sección se publican artículos generados en la Facultad de Diseño, relacionados con las actividades de docencia, extensión, formación en investigación o internacionalización, las cuales son reflejo de la dinámica y de las actividades realizadas por docentes, estudiantes y egresados; esta sección no puede superar el $20 \%$ del contenido.

Textos. En esta sección se publican reseñas, traducciones y memorias de eventos relacionados con las publicaciones en Arquitectura y Urbanismo.
A Frecuencia de publicación

Desde 1999 y hasta el 2015, la Revista de Arquitectura (Bogotá) publicó un volumen al año, a partir del 2016 se publicarán dos números por año en periodo anticipado, enero-junio y julio-diciembre, pero también maneja la publicación anticipada en línea de los artículos aceptados (versión Post-print del autor).

La Revista de Arquitectura (Bogotá) se divulga mediante versiones digitales (PDF, HTML, EPUB, XML) e impresascon un tiraje de 700 ejemplares, los tiempos de producción de estas versiones dependerán de los cronogramas establecidos por la editorial.

Los tiempos de recepción-revisión-aceptación pueden tardar entre seis y doce meses dependiendo del flujo editorial de cada sección y del proceso de revisión y edición adelantado.

Con el usuario y contraseña asignados, los autores pueden ingresar a la plataforma de gestión editorial y verificar el estado de revisión, edición o publicación del artículo.
(A) Canje

La Revista de Arquitectura (Bogotá) está interesada en establecer canje con publicaciones académicas, profesionales o científicas del área de Arquitectura y Urbanismo, como medio de reconocimiento y discusión de la producción científica en el campo de acción de la publicación.

Mecanismo

Para establecer canje por favor descargar, diligenciar y enviar el formato: RevArq FP20 Canjes

Universidad Católica de Colombia
(202I, enero-junio). Revista de
Arquitectura (Bogotá), 23(I),
I-I24. Doi: 10.14718

ISSN: I657-0308
E-ISSN: 2357-626X
Especificaciones:
Formato: $34 \times 24 \mathrm{~cm}$
Papel: Mate II5 g
Tintas: Negro y policromía

Contacto

Dirección postal:

Avenida Caracas No. 46-72

Universidad Católica de Colombia

Bogotá D. C., Colombia

Código postal: 111311

Facultad de Diseño

Centro de Investigaciones (CIFAR)

Sede El Claustro. Bloque "L", 4 piso

Diag. 46A No. 15b-10

Editor, Arq. César Eligio-Triana

Teléfonos:

+57 (1) $3277300-3277333$

Ext. 3109; 3112 o 5146

Fax: +57 (1) 2858895
Correo electrónico:

revistadearquitectura@ucatolica.edu.co cifar@ucatolica.edu.co

Página WEB:

www.ucatolica.edu.co

Vínculo Revistas científicas

http://publicaciones.ucatolica.edu.co revistas-cientificas

https://revistadearquitectura.ucatolica.edu.co/ 
Reflexiones en torno a la enseñanza de la arquitectura y el urbanismo en Colombia. Conversaciones con Juan Carlos

m. Pérgolis Valsecchi, René Carrasco Rey y Juan Carlos del

¿ Castillo

Reflections on the teaching of architecture and urban planning in Colombia. Conversations with Juan Carlos Pérgolis Valsecchi, René Carrasco Rey y Juan Carlos del Castillo

웅

La experiencia de caminar en ciudades latinoamericanas

The experience of walking in Latin American cities

Movilidad urbana de la población en la ciudad de Encarnación * Paraguay. Desarrollo urbano y gestión ambiental

$m$ Urban mobility of the population in the city of Encarnación, Paraguay.

这

ษั

La vivienda adecuada financiada según el ingreso.

El discurso de las Naciones Unidas

பं

La arquitectura frente a las innovaciones pedagógicas.

Pervivencia y resignificación de la Escuela Nueva en el Cono Sur Architecture facing the pedagogical innovations. Survival and resignification of the New School in the Southern Cone

Aporte de las competencias investigativas a la integración de saberes curriculares.

Una visión en el escenario de aprendizaje del diseño

arquitectónico

Contribution of research competencies to the integration of curricula knowledge. A vision in the architectural design learning scenario

Adobe como saber ancestral usado en construcciones

N autóctonas de Pore y Nunchía, Casanare (Colombia)

j. Adobe as an ancestral knowledge used in autochthonous constructions $\stackrel{4}{\longleftarrow}$

Paisaje construido y sustentabilidad urbana: huellas

$œ$

El Plan de Transformación de Osorno

Built landscape and urban sustainability: Identity prints of the modern landscape. The Osorno Transformation Plan

Indicadores de sostenibilidad social y su relación con el

$\hat{a}$

ن்

노 caso de estudio en Cuenca (Ecuador)

ن

Confort térmico en vivienda social multifamiliar de clima cálido en Colombia

느 Thermal comfort in multi-family social housing in a warm climate in ن் Colombia
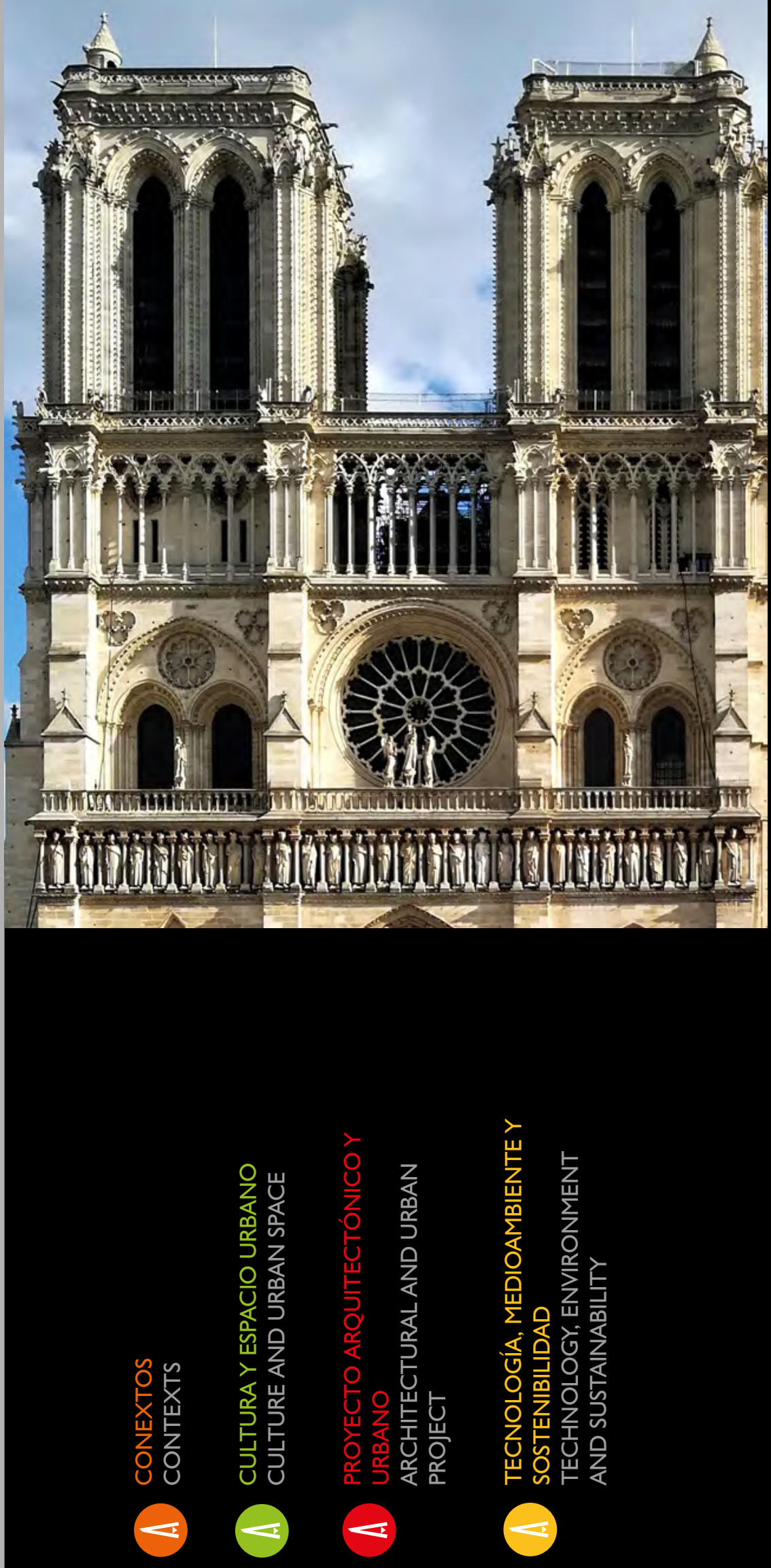

La Revista de Arquitectura es de acceso abierto, arbitrada e indexada y está presente en:
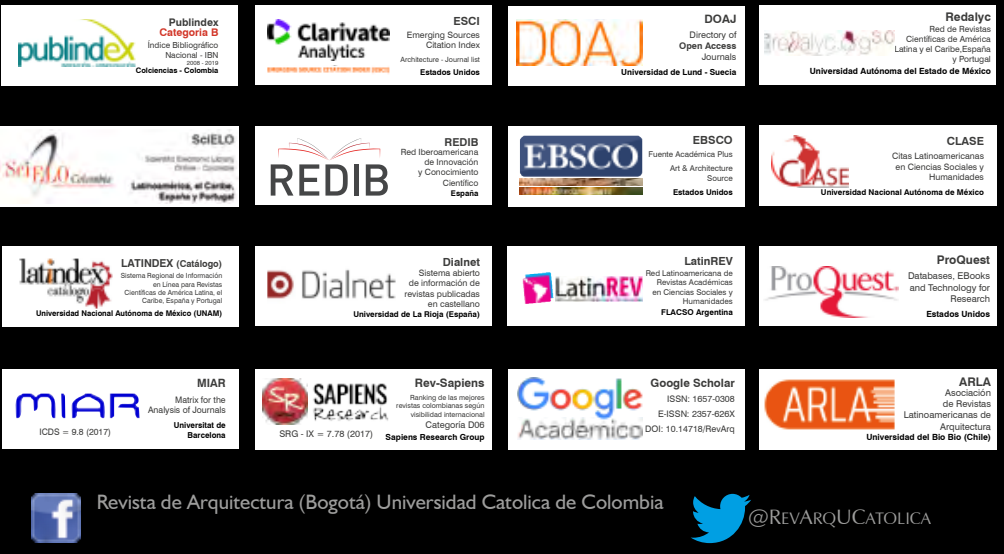

8
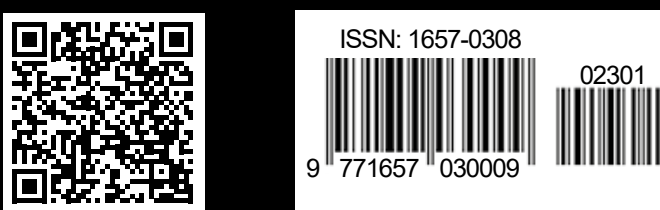